\title{
BIBLIOTEQUES PARTICULARS GIRONINES DEL SEGLE XV
}

\author{
Enric Mirambell i Belloc
}

El segle xv té una gran transcendència en la història del llibre. La invenció de la impremta i la seva propagació representa no tan sols assolir una fita tecnològica, sinó que és la resposta a la demanda de llibres, fruit d'un moment en què la cultura va deixant de ser patrimoni d'unes minories, i especialment de l'estament clerical, i va estenent-se a camps cada vegada més amplis i més variats.

-L'estudi de les biblioteques particulars que existien resulta ben alliçonador per comprendre aquest ambient cultural que s'anava desenvolupant. En l'estudi present pretenc donar una visió de conjunt de les biblioteques que la documentació ens permet conèixer. Unes ja publicades anteriorment, i altres que ara presentem per primera vegada.

A la Girona medieval existien les biblioteques de la Catedral, de la Col-legiata de Sant Feliu i les dels diversos monestirs i convents, entre les quals destacaven la dels benedictins de Sant Pere de Galligants i la dels predicadors del convent de Sant Domènec.

La Biblioteca de la Catedral, la va estudiar magistralment el doctor Lluís BATLle I Prats', que també ens ha donat alguna

I La Biblioteca de la Catedral de Gerona desde su origen hasia la imprenta, CSIC, Instituto de Estudios Gerundenses, 1947. 
notícia sobre la de Sant Feliu ${ }^{2}$, la de Sant Pere de Galligans ${ }^{3}$ i la dels dominics ${ }^{4}$.

Sobre la de Sant Feliu han publicat també JANINI i MARQUES Planagumả, Francesc Xavier Altés, i Tomás Noguer ${ }^{5}$.

De les restants, en sabem poca cosa més que el que ens en diuen el pare Villanueva i els continuadors de La España Sagrada.

Les biblioteques particulars de què tenim notícia, corresponents al segle xv, no totes van pertànyer a homes d'Església, sinó que algunes eren propietat de seglars de diverses professions. A les subhastes de llibres sabem que també hi van concórrer homes de professions lliberals i fins i tot algun artesà, fet molt representatiu dels nous corrents per on anava la vida cultural.

Fins ara la documentació ens permet donar notícia de deu biblioteques particulars de considerable importància per la quantitat i la qualitat dels seus fons, i d'un nombre més crescut de petites col-leccions bibliogràfiques.

Les quatre biblioteques que ara publiquem per primera vegada van pertànyer a l'escrivà Raimond Renard, que va ser inventariada l'any 1428, al jurista Antoni Agullana, documentada l'any 1445, la del preposit de la Pia Almoina del Pa de la Seu, Narcís Bruguera, inventariada l'any 1480, i la del prevere de Capítol, Bartomeu Oristà, inventariada l'any 1488. De totes quatre, en transcriurem en l'apèndix els documents que ens les descobreixen. Algun d'aquests documents, el transcrivim íntegre, mentre que altres només en el que fa referència als llibres.

El doctor [Batlle i Prats] en tres dels seus treballs sobre temes

2 "La biblioteca de la colegiata de San Félix de Gerona», Gesammelte... Kulturgeschichte Spaniens 21, Münster, 1963, pp. 146-148.

3 "La Biblioteca del Monasterio de San Pedro de Galligans", Revista de Gerona 35, 1966, pp. 28-29.

4 «Encuadernaciones y libreria del convento de Santo Domingo de Geronas, Revista Bibliográfica y Documental 2, abril-juny 1947, pp. 269-277.

5 Janini, J. Marqués, J.M., «Manuscritos de la Colegiata de San Félix de Gerona», Hispania sacra 15, 1962.

Aıtés i Aguno, Francesc Xavier, «Llibres retrobats de la Col-legiata de Sant Feliu de Gironan, AIEG XXIV, 1978, pp. 37-56.

Noguer Musqueras, Tomás, «La Biblioteca de la Colegiata de San Félix de Gerona», Revista de Gerona 35, 1966, pp. 42-46. 
bibliogràfics ens parla de col-leccions de llibres corresponents al segle $x v^{6}$.

La primera, atenent l'ordre cronològic, és la biblioteca del cardenal Anglesola, que va ser bisbe de Girona de l'any 1384 al 1408. L'any 1406, en ocasió de preparar l'estatge per al rei en el palau episcopal, va manar que es col-loqués la biblioteca en tres caixes i que es diposités a la tresoreria de la seu. Al mateix temps disposava que es redactés un inventari dels llibres que la integraven. En aquest inventari es descriuen minuciosament quarantados llibres, la majoria de tema jurídic.

En un altre dels esmentats treballs, el doctor [Batlle] ens dóna notícia d'inventaris, testaments i encants en què queden reflectits els fons bibliogràfics que posseïen alguns servidors de la Catedral: beneficiats, preveres de Capítol, canonges, etc. Entre aquests, al segle xv corresponen el canonge Berenguer de Fluvià; el pavorde del mes de gener i de l'Almoina del Vestuari de la Seu, Bernat Vilar; el claver, Pere Olivera, els beneficiats Ramon Tirany, Pere Mata, Bernat Baiuli, Vicens Calabuig i Francesc Padret; el canonge Antoni Roca, el rector de l'hospital de la Seu Guillem Riba, l'ardiaca major Dalmau de Raset, els beneficiats Joan Sunyer, Marc Sabater, Jaume Bagó i Pere Pin; i el claver Bernat Castellar.

Els documents que es refereixen a aquestes col-leccions de llibres corresponen als anys que van del 1410 al 1466.

Entre elles no hi ha cap biblioteca notable; però mentre que algunes disposen d'uns pocs llibres, altres ja arriben a la vintena, nombre ja considerable, si tenim en compte el valor i la raresa del llibre en aquelles dates. En general, la temàtica és la que s'escau tractant-se d'homes d'Església: llibres litúrgics i de devoció, tractats de Teologia i de Filosofia, i alguna obra històrica o literària.

6 "Inventari dels llibres de Berenguer d'Anglesola», dins Estudis universitaris catalans, vol. XVII, Barcelona 1932, pp. 234-249.

«Noticias de libros antiguos de servidores de la Catedral de Gerona, 1335 1594n, Hispania sacra 22, 1969, pp. 425-466.

«La biblioteca del mestre Marc Roca, metge de Girona, 1427», Tercer congrés d'historia de la medicina catalana, vol. I, Lleida 1981, pp. 11-21. 
També el doctor BATLLE, en col-laboració amb el doctor MiLLẢs, ens fa conèixer les col-leccions de llibres que posseïen els jueus gironins dels primers anys del segle $x^{7}$. Encara que els llibres que figuren en aquests inventaris són exclusivament referents a la religió jueva, no deixen de ser un signe del nivell cultural d'un col-lectiu gironí, almenys pel que fa a la religió que professaven.

En el protocol del notari Escuder, hi trobem un volum dedicat totalment als afers derivats del testament de l'escrivà de Girona Raimon Renard ${ }^{8}$. El llibre conté el testament, l'inventari i l'encant dels béns de l'esmentat escrivà, i tota una sèrie de documents relacionats amb l'actuació dels marmessors. Un estudi exhaustiu d'aquest volum notarial seria ben interessant; però de moment m'he limitat al que pertoca als llibres que posseïa l'esmentat Raimon Renard.

En el moment en què atorga el testament estava casat amb una muller anomenada Margarida, i no tenia fills; però en determinar l'ordre d'herència esmenta els fills que en un futur li puguin néixer.

No disposa de gaires llibres, però són molt variats quant a les matèries, la qual cosa ens presenta una personalitat d'àmplia cultura, que no s'acontenta solament amb els coneixements professionals.

Una bona part dels llibres que componen aquesta biblioteca és de tema religiós: Evangelis, Epístoles, Proverbis, Psalms, Breviari, Hores de Santa Maria, el Vita Christi, Vides de Sants. Però també hi trobem les Cròniques de Sant Isidor, el Llibre de les meravelles de Ramon Llull, el De consolatione de Boeci, el Llibre dels Àngels d'Eiximenis. I llibres professionals, entre els quals destaca un tractat de l'art de notaria.

La descripció d'aquests llibres que es fa en l'inventari és molt sumària, encara que, en general, és suficient per la identificació

${ }^{7}$ Millas Vallicrosa, J.M.; Batlle Prats, Lluís, «Inventaris de llibres de jueus gironins", Butlleti de la Biblioteca de Catalunya, v. VIII, 1928-1932 (1934), pp. 5-45.

${ }^{8}$ Apèndix documental, I. 
de les obres. En canvi, fa una detallada descripció externa dels llibres, concretant els materials, la mida, l'enquadernació i fins i tot el color de les cobertes.

Tots aquests llibres eren manuscrits i alguns exemplars eren molt valuosos, com podem comprovar pel preu que se'ls adjudicà en la subhasta.

També em sembla remarcable el fet que el comprador d'un dels llibres fos de professió assahonador, la qual cosa ens mostra que l'interès pels llibres en aquell moment ja no es concretava en unes determinades classes, sinó que afectava fins i tot algun artesà.

Antoni Agullana, doctor en Lleis, ciutadà de Girona, va estar casat en primeres núpcies amb Margarida Guixeras, i en segones núpcies amb Leonor. En morir, institueix hereu universal dels seus béns Miquel, fill del segon matrimoni.

Miquel, l'hereu, paga a la seva mare, Leonor, 500 florins per raó de la seva dot, i el pagament el fa amb llibres. També paga 200 florins de llegítima, igualment en llibres, a Antoni Agullana, clergue, doctor en drets, fill del primer matrimoni.

Els documents que s'atorguen per fer aquests pagaments corresponen a l'any $1445^{\circ}$. En ells es relacionen dinou llibres que dóna a Leonor, i deu llibres que dóna a Antoni.

En la relació consta el preu que s'assigna a cadascun dels llibres. Encara que per la descripció summament simple que es fa dels llibres ens resulta dificil calcular-ne el seu valor, em sembla que, el que se'ls atribueix, el podem considerar extraordinàriament elevat.

Tots aquests llibres eren manuscrits, i formaven part de la Biblioteca del jurisconsult Antoni Agullana.

És la biblioteca professional d'un jurisconsult; tots els llibres són de matèria jurídica. Hi trobem els tractats de Baldus de Perusio, d'Antonius de Butrio, de Dominicus de Sancto Gemminiano, de Johannes Calderinus, i els textos més representatius del dret civil i canònic.

La biblioteca de Roger de Cartellà, que coneixem pel testa-

9 Apèndix documental, II i III. 
ment d'aquest clergue gironi ${ }^{10}$ membre d'una notable familia, propietària del castell de Cartellà, era d'una gran riquesa. Va deixar una part de la biblioteca a la Catedral, una altra part, a la col-legiata de Sant Feliu, i alguns llibres a les esglésies parroquials de Burbáguena i Cosuenda, del bisbat de Saragossa.

La biblioteca de Roger de Cartellà estava integrada per un considerable nombre de llibres jurídics; però també hi trobem esmentat un exemplar del Talmut, el Libre disputationum iudeorum de Petrus Alfonsus, obres de Sant Isidor, un Psaltiri amb glosses de Nicolaus de Lyra, diversos llibres de Sermons, un d'ells de Sant Vicens Ferrer, obres de Dionís el Cartoixà, un exemplar de les Constitucions de Catalunya, la Civitas Dei de sant Agusti, i altres obres especificament religioses.

Narcís Bruguera, prepòsit de la Pia Almoina, en el seu testament, atorgat davant el notari Pere Escuder, institueix hereva dels seus béns la institució de la qual era prepòsit. Després de la mort del testador, el seu succesor en el càrrec, Pere Ramon Mut, rep dels marmessors de Bruguera els llibres que havien integrat la seva biblioteca particular i que van passar a ser propietat de la Pia Almoina".

Aquesta biblioteca és realment copiosa i variada en matèries. En el preàmbul del document ja ens parla de llibres jurídics, de Medicina i de Gramàtica. I en la relació estableix dos apartats: llibres jurídics i llibres no juridics. Dins el primer apartat s'inclouen 39 llibres i dins el segon, 85 llibres.

Entre els llibres jurídics hi ha aquells tractats i textos corrents, que també trobem en les biblioteques de Raimon Renard i d'Antoni Agullana. Però la col-lecció de llibres jurídics de Bruguera és més rica que les altres esmentades. Entre altres, hi trobem obres de Bartholomeus de Saxoferrato, Johannes Fabri, Angelus d'Arescio, Johannes de Andree, Petrus de Bracha, Dominicus de Sancto Gemminiano.

Entre els llibres no jurídics hi abunden obres de Gramàtica,

${ }^{10}$ Mirambell Belloc, E., "El testamento de Roget de Cartellá y su notable bibliotecan, AIEG, XVII, 1964-1965, pp. 383-403.

"Apèndix documental, IV. 
de Medicina, de Filosofia. Obres d'Aristòtil, Alexandre de Vailladei, Boeci. I un bon nombre d'exemplars de Teologia, de Biblia, i de Moral.

La descripció dels llibres és ben simple pel que fa a l'autor i el títol, i en canvi es deté més en la presentació externa. Especifica sempre si són escrits sobre pergami o sobre paper, la qualitat de l'enquadernació, el color de la pell que recobreix les cobertes; el nombre de tanques que porten i si n'hi falta alguna. En canvi, no ens indica mai si es tracta de manuscrits o d'impresos; en aquella data (1480), ja hi podia haver algun exemplear imprès.

Encara que no hi consta el valor pecuniari dels exemplars, no hi ha cap dubte que es tracta d'una biblioteca molt valuosa, tant pel nombre com per la varietat temàtica dels llibres que la integraven.

La biblioteca del cardenal Margarit, l'hem de considerar importantíssima. Coneixem el seu contingut per dos documents: un és de l'any 1484, el mateix any de la mort del Cardenal, i es tracta d'un memorial datat a Roma, en el qual Margarit dóna instruccions sobre la destinació dels béns que havia deixat en terres gironines. I d'una manera molt detallada s'hi inclouen els seus llibres ${ }^{12}$. L'altre ja és de l'any 1509 i és un inventari de llibres que havien pertangut al cardenal i que van ser subhastats. EI van publicar Rubió i Madurell en la seva obra monumental sobre els impressors primitius de Barcelona ${ }^{13}$.

A la biblioteca de Margarit hi havia exemplars ben notables. En destaca un Ptolomeu, que, presentat amb tots els honors, en fa obsequi al rei Ferran el Catòlic. També s'hi trobaven les obres bàsiques de la cultura medieval, com són les Etimologies de sant Isidor, els llibres d'Història de Paulo Orosio i d'Eusebi de Cesàrea, obres de sant Jeroni, sant Gregori, sant Bernat, sant Joan

12 Mirambell Belloc, E., "Un memorial del cardenal Margarit», dins IEG, XXII 1974-1975, pp. 75-96.

13 Madurell Marimon, José M., Rubio i Balaguer, Jorge, Documentos para la historia de la imprenta i libreria en Barcelona (1474-1553), Barcelona 1955, doc. 274 , pp. $488-500$. 
Crisòstom, sant Ambròs, diversos tractats teològics, bíblics i un bon nombre de llibres de Dret canònic.

Alguns són rics exemplars en pergamí, decorats amb or i policromia. En el Memorial valora el conjunt dels llibres en 1.857 florins d'or, i a algun exemplar assigna la substanciosa quantitat de cent florins.

Una altra biblioteca gironina que sens dubte degué ser molt important és la que va pertànyer al Vicari General i home de confiança del Cardenal Margarit, el canonge Andreu Alfonsello. Però només la podem conèixer per referències, sense que haguem pogut veure cap relació dels llibres que la integraven. En el testament d'Alfonsello, que he estudiat ${ }^{14}$, hi ha una clàusula en què deixa la seva biblioteca particular a la de la catedral d'Elna. Diu que lliurarà al notari la relació dels llibres. Però el mateix notari al peu del document anota que no se li va lliurar la relació esmentada. La importància d'aquesta biblioteca, la podem deduir de la personalitat rellevant del seu propietari, i del que disposa que per rebre aquest llegat el local de la Biblioteca de la Catedral d'Elna haura de ser reformat i ampliat; s'hi hauran de construir noves prestatgeries, de la manera que ell mateix indica.

Finalment ens ocupem dels llibres del prevere de Capítol, Bartomeu Oristà, que consten en l'inventari realitzat en ocasió de la seva mort, l'any $1488^{15}$.

L'inventari ens descriu la casa que habitava l'esmentat prevere, situada prop del portal de sant Cristòfol, i de la torre Gironella. És difícil precisar l'emplaçament exacte d'aquest habitatge; però sembla que el podriem fixar entre el portal de sant Cristòfol i el lloc on més endavant s'establí la caserna dels alemanys. Per la seva situació tan propera a la muralla, en un lloc molt afectat per les contínues obres de defensa de la ciutat, és probable que aquesta casa desaparegués en un canvi de configuració de l'indret.

14 Mirлmiı Bisıx, E., «El testament d'Andreu Alfonsello, vicari del Cardenal Margarit», Revista de Girona 82, 1978, pp. 9-12.

15 Apèndix documental, V. 
Bartomeu Oristà era un clergue al servei de la catedral, i en la descripció de la seva biblioteca trobem dues obres de les quals ell era l'autor. Una d'elles és un llibre de sermons per ser recitats a la capella papal, i l'altra, un Repertorium. La primera ens pot fer suposar que es tractava d'un predicador de cert prestigi.

Els llibres que componen aquesta biblioteca són essencialment de tema jurídic, com correspon a un doctor en drets, i de tema religiós, adients a la seva condició d'eclesiàstic. També hi trobem alguns llibres d'història, com és el De bello judaico, de Flavio Josefo i les Gestes dels romans de Polibi. També hi figuren diverses obres de Ciceró.

Els llibres es trobaven a l'interior de tres caixes; però no veig que això obeís a cap raó sistemàtica.

La descripció de les obres és molt sumària, i presenta errors ortogràfics, cosa que en dificulta la identificació.

Amb tot, es pot tenir una idea general del contingut d'aquesta biblioteca, composta per prop de mig centenar de llibres.

Per la data de l'inventari és possible que alguns dels llibres fossin impresos; però això no s'especifica. En canvi, sí que, com hem vist en altres casos, es fa esment del tipus d'enquadernació i de la mida de cada exemplar.

El nombre relativament elevat de biblioteques particulars de què hem pogut trobar notícia documental és un signe de l'evolució cultural que es va experimentar en aquell segle, que contempla el pas de l'Edat Mitjana al Renaixement.

El llibre ja no és solament patrimoni de catedrals i monestirs, sinó que també el trobem en les cases particulars, de clergues, de jurisconsults, de metges, d'escrivans i fins i tot d'algun artesà. Prova evident que la Cultura està ampliant considerablement el seu camp. 


\section{APÈNDIX DOCUMENTAL}

Inventari

(fol. 13)

29 juliol 1428

Item un libre de paper ab cisterns de pergamins appellat de Vita Christi, ab tencadors d'argent ab cubertes de post.

Item altre libre de paper ab cubertes de posts ab pell vermeya $e$ bulletes intitulat libre de moretes.

Item altre libre de pergami intitulat proverbiorum seu parabole Salomonis cum postibus cohopertis de pelle viridi.

Item altre libre de paper ab cubertes de pergamí a un ha Evangelis.

Item altre libre de paper ab cubertes de pell verda ab correx vermey qui comensa libre de la alta saviesa.

Item altre libre de paper de forma menor ab posts cubertes de pell vermeya e bulletes appellat Boeci de consolacio.

Item altre libre de paper ab cap coerns de pergami qui comensa puisque lo primer para del humanal linatge cubert de pell blanca.

Item altre libre de paper qui comensa en tristicia e appellees Libre de meravelles, cubert de pell verda.

Item altre libre de forma menor de paper intitulat Caroniques de Sant Isodorus ab cubertes de pell blanca e correx negre.

Item altre libre de forma menor de paper qui comensa Rubrica de la Biblia lo testament veyl ab cubertes vermeyes e correg.

(fol. 15)

Item un libre de Constitucions escrit en pergamí ab posts cubertes de pell vermeya.

Item un libre de pergamí ab posts cubertes de pell vermeya que comensa incipiunt vite sanctorum.

Item un saltiri scrit en pergamí.

Item un libre dels angels en paper ab posts cubertes de pell blava.

Item unes Hores de Sancta Maria ab salms de Domine ne.

(fol. 18)

Item un libre lo qual comensa Bonifacius episcopus cubert de posts ab pell blava.

Item un libre sisternat de pergamí lo qual comensa frare Ambros cubert ab posts de paper ab pell vermeya $a b$ una correge.

Item altre libre de pergamins tancretus qui comense incipit ordo iudiciarius, cubert de posts ab pell vermeya descolorada ab tencadors. 
Item altre libre lo qual es test de logique veya cubert de posts ab tencadors cubert de pell verda.

Item un breviari de pergamins cubert de posts ab pell verda descolorida.

Item un libre descornat ab post ligat ab cordes lo qual no sabem que es.

Item un tractat de l'art de notaria de pergamí.

Item un tractat de summa de Rolandi.

\section{Encant}

(fol. 41)

Item un saltiri scrit en pergamins an Johan Scuder not.

Item unes hores de Sancta Maria ab salms de Domine an Jac. Adolf.

Primo un libre de Moyses de paper en romans an Ricart assahonador

11l. $\mathrm{X}$ s. III

Item un libre dels angels a mossen Pere

Fiter sacristà de Bescanó

Item un Boeci de consolació en romans de paper a mossen Bernat Jover

Item altre llibre ab tancador dargent en romans ab evangelis epistoles, en Francesc Grau

Arxiu Històric Provincial de Girona, protocol del notari J. Escuder, notaria 8, núm. 13, any 1428.

Llibre que conté el testament, inventari, comptes i altres documents relacionats amb l'actuació dels marmessors de l'escrivà de Girona en Raimon Renard.

\section{II}

Elienor uxor honorabilis Anthonii Agullana quondam legum doctoris civis Gerunde tenens et posidens hereditatem et bona que fuerunt dicti quondam viri mei pro dote spontalitio et aliis iuribus et cetera. Confiteor et recognosco vobis Petro Michaeli Agullana filio michi et dicto quondam viro meo comuni heredique universali dicti viri mei priusquam presenti quod vos insolutum pro rata dotis me ac aliorum iurium michi pertinentium in bonis dicti quondam viri mei dedistis michi egoque a vobis eodem nomine confiteor habuisse numerando quingentos florenos auri Aragonum prefatos autem quingentos florenos confiteor habuisse in hunc modum nam pro ipsorum quingentorum fflorenorum valore recepi libros sequentes scilicet: 
Unum Decretum pro centum florenis

C flor.

Item alium librum vocatum Ardiacha sobre lo sizé

V flor.

Item alium vocatum lo baldó sobre l'ignocent pro decem flor.

$\mathrm{X}$ flor.

Item alium vocatum lo baldó sobre lo quart e scilicet sobre lo quint pro viginti flor.

$\mathrm{XX}$ flor.

Item alium librum vocatum los dos repertoris de calderi pro viginti florenis

$\mathrm{XX}$ flor.

Item alium librum vocatum digesta vella pro quindici flor.

XV flor.

Item alium librum vocatum lo baldo sobre lo seté pro X florenis

$\mathrm{X}$ flor.

Item los bartols sobre la digesta vella pro decem octo florenis

Item quondam librum vocatum lo baldo sobre la segona part de la digesta vella pro decem flor.

Item lo baldo sobre lo VIII e VIIII pro duodecim flor.

Item scilicet sobre lo terc pro decem flor.

Item scilicet sobre lo primer e segon del Codi pro quindecim flor.

Item baldo sobre lo primer e segon del Codi pro XVIII flor.

Item alium librum vocatum repertori de butrio pro XXX flor.

Item alium vocatum baldo sobre lo terç pro $\mathrm{X}$ flor.

Item los butrions en sis volums pro CXXXX flor.

Item encara sobre lo terç pro $\mathrm{XXX}$ flor.

Item unes repeticions de canones pro XV flor.

Item un Codi bo pro duodecim florenis

XVIII flor.

$\mathrm{X}$ flor.

XII flor.

$\mathrm{X}$ flor.

XV flor.

XVIII flor.

XXX flor.

$\mathrm{X}$ flor.

Et ideo renunciando de dictis quingentis florenis per vos mihi in modum predictum solutis hanc vobis facio apocam de soluto salvo tamen michi iure in residuo. Testes Monserratus Robert textor pani lini et Bernardus Oliveres clericus Gerunde. Actum Gerunde die lune XXVI die aprilis anno predicto XLV

(Notari Miquel Pere; notaria núm. 1 de Girona; núm. 246; anys 1444-1445) 
Petrus Citjar notarius civis Gerunde procurator ad hec et alia eciam cum libera et generali administratione legitime constitutus ab honorabili Anthonio Agullana clerico decretorum doctore filio honorabilis Anthonii Agullana quondam legum doctoris civis Gerunde et domine Margarite quondam prime uxoris sue prout de huiusmodi procuratione et potestate plene constat publico instrumento acto in posse subscripti notarii quartadecima die aprilis anno a nativitate Domini $\mathbf{M}^{\circ}$ CCCC XXX sexto. Gratis dicto nomine absolvo, diffinio penitusque remitto vobis Petro Michaeli Agullana filio dicti honorabilis Anthonii Agullana et domine Elionoris viventis que fuit uxor eiusdem heredique universali cum beneficii inventarii dicti quondam patris vestri presentis et vestris et cetera totam partem hereditatem et legitimam dicti Anthonii Agullana principalis mei paternam et maternam et aliud quodvis ius quod dicto meo principali pertinet et pertinere potest et debet in et super hereditatibus et bonis que fuerunt dictorum honorabilium Anthonii Agullana et domine Margarite coniugum parentium ipsius principalis mei et cetera in et super bonis que fuerunt Gregorii Guixeres avi et Johanis Guixeris avunculi dicti mei principalis maternorum quibusvis rationibus sive causis ita et cetera facio finem et cetera sicut et cetera cedendorum quibus et cetera pro his absolutione deffinitione remissione et fine et recepisse ducentos florenos auri de Aragonia quos dictus defunctus dicto principali meo legavit in eius ultimo testamento et quia obcionem dedit eidem meo principali quod pro illis reciperet libros iuris extimatos usque ad ipsam quantitatem ego dicto nomine pro ipsorum $\mathrm{CC}$ florenorum valore accepi libros sequentes scilicet:

Quendam librum vocatum Innocent pro sex ff. : VI ff. Item quendam librum vocatum Rosari pro triginta ff. : XXX ff. Item alium librum vocatum Speculador pro XXX ff. : XXX ff. Item alium librum vo. Baldo super ffeudis pro $X \mathrm{ff}$. $X$ ff. Item alium librum vo. Addiciones Iohannis Andree pro XX ff.

Item duos libros vo. Gemininas pro XXX ff.

Item alium librum vo. Baldo super Digesto veteri pro XXV ff.

$: \mathrm{XX}$ ff.

: XXX ff.

Item alium librum vo. Summa hostiensis pro XXV ff. Item alium librum vocatum Volum pro XII ff. Item alium librum vocatum Sisen pro XII ff.

: XXV ff. : XXV ff. : XII ff. : XII ff.

Et ideo renunciando et cetera si plus et cetera promissi et iuravi et cetera salvo temen et reservo dicto meo principali hec que dictus pater suus in dicto testamento ultra dictos $\mathrm{CC}$ florenos voluit eundem meum principalem havere ut sequitur. 
Et primo quod si dictus principalis meus voluerit facere suam habitationem dicti patris sui una cum honorabili domina Elienore vivente que fuit uxor eiusdem et aliis infantibus suis dimisit ipsi meo principali cameram maiorem prope la sala dicti hospitii ad suum adinprivium.

Item voluit quod vivente dicta domina Elienore uxor sua serva et alii servitores dicte uxori sue ac qui erunt in dicta domo sua teneantur servire ipsi meo principali ad coquinandum et alias voluit etiam quod dum dictus meus principalis in dicta domo erit sibi detur vinum tam album quam virmilium per heredem ipsius testatoris. Et voluit etiam quod totiens quotiens dictus meus principalis voluerit ire ad vineam dicti defuncti habeant illich ipsi et eius servitores ademprivium ex ffructibus et ffruiters et vino pro dispendio et usu ipsius et illorum quos secum illuc ducet. Hec enim ultra dictos $\mathrm{CC}$ ff. dicto meo principali legata retineo salvo et reservo dicto meo principali. Deinde salvo et reservo ipsi meo principali ius future successionis si quod forte sibi pertineret in et super bonis que fuerunt dicti patris sui.

Et sic salvis etcetera promissi et iuravi hec igitur etcetera.

Et ego dictus Petrus Michael Agullana predictis omnibus consentio et fateor dictos libros extimatos fuisse pecunie summis ac modo et forma superius expressatis et promissi et iuravi non contrafacere vel venire ratione minoris etatis et cetera immo minor XXV maior vero XVI annis et cetera. Ad hec ego Elienor uxor dicti honorabilis Antonii Agullana tenens et posidens etcetera confiteor vobis dicto discreto Petro Citjar dicto nomine quod de voluntate et assensu meis pro satisfactione dictorum $\mathrm{CC}$ fflorenorum vobis traditi fuerunt extimati extimationibus predictis libri predicti numero decem ut superius continetur et promissi et iuravi non contraire et cetera.

Actum est hoc Gerunde die lune XXVI aprilis anno predicto Testes firme dictorum Petri Citjar et Petri Michaelis Agullana sunt venerabilis Petrus de Scala civis Gerunde, Bartholomeus de Avellaneda domicellus domus castri de Orriollis, Petrus Lombardi et Michael Vinyals scriptor Gerunde. De firma dicte domine Elienoris que premissis consentit eaque laudavit et firmavit dicta die sunt testes Montserratus Robert et Anthonius Bruguera textores pani lini Gerunde.

(Notari Miquel Pere, notaria 1 de Girona, núm. 426)

\section{IV}

Sit omnibus notum quod ego Petrus Raimundi(sic) Mut presbiter praepositus Elemosine Panis Sedis Gerunde quam Elemosinam honorabilis dominus Narcissus Bruguera quondam prepositus precessor meus in eadem cum suo ultimo testamento quod ffecit et otorgavit in posse discreti Petri Escuder notarii Gerunde die et anno in eo conten- 
tis sibi herede instituit. Gratis et ex certa scientia conffiteor et recognosco vobis discretis dominis Ffrancisco Miralles et Poncio Traver presbiteris benefficiatis in dicta sede manumissoribus et executoribus dicti ultimo testamenti dicti defuncti presentibus quod delliberastis et realiter michi tradidistis libros iuridicos et de medicina et de Gramatica et alios qui erant et sunt de dicta hereditate et manumissoria qui et prout inferius sunt descripti.

Primo 1 libra scrit en pergami ligat de posts ab pell vermella aquinsada ab quatre tencadors manchan y tres appellat VI liber Decretalium.

Item 1 libra scrit en pergami ligat de posts ab pell vermella apellat digesta nova a poc valor.

Item altra libra scrit en pergamí ab quatra tancadors e cobert de pell vermella apellat digesta myllor de l'altra.

Item altra libra scrit en pergamí ligat de posts ab pell vermella e verda appellat volumen.

Item altra libra scrit en pergamí ligat de posts ab pell verda ab quatre tencadors appellat codi.

Item altra libra scrit en pergamí ligat de posts ab dos tencadors appellat inforcada, bo.

Item altra libra scrit en pergami ligat de posts ab pell verda qui ha quatre tencadors ha ni hu appellat digesta vella.

Item altra libra scrit en pergami ligat de posts ab dos tencadors ab pell d'aluda appellat ignoscent.

Item altra libra scrit en paper ligat de posts sens pell ab quatre tencadors appellat Angelus de Arescio super titulandi actionibus iustitiae.

Item altra libra scrit en pergamí ligat de posts ab quatre tencadors la-1 mancha appellat Enrichus Bosch super I et II decretalium.

Item altra libra scrit en paper ligat de posts ab pell blancha be squinsada ab quatre tencadors manchan y tres appellat $\mathrm{P}^{\circ}$, Jacobi.

Item altra libra scrit en paper ligat de posts ab quatre tencadors manchant y hu appellat Bartholus super prima parte digestus veteris.

Item altra libra scrit en paper ligat de posts ab quatre tencadors ab pell vermella e negra appellat Johannes Fabri super iustitia.

Item altra libra scrit en paper ligat de posts sens pell ab quatre tencadors appellat Angelus de Aressio super titulo de apellationibus digesti novi.

Item altra libra scrit en paper ligat de posts ab quatre tencadors manchant y dos appellat Bartholomeus super secunda parte digesti novi.

Item altra libra scrit en paper ligat de posts sens pell ab dos tencadors appellat novellae Johanis Andree.

Item altra libra scrit en paper ligat de posts ab pell blanca ab quatre tencadors manchant ni dos appellat spicha Petri de Bracha.

Item altra libra scrit en paper ligat de posts ab pell groga ab quatre tencadors manchant hi tots appellat Spicha sseculi. 
Item altra libra scrit en paper ligat de posts sens pell ab quatre tencadors appellat Dominicus de Sancto Gemminiano super III et IIII, VI decretalium.

Item altra libra scrit en paper sisternat de pergamí ligat de posts sens pell ab quatre tencadors appellat Quiricus Bosch super III decretalium.

Item altre libra scrit en paper sisternat de pergamí ligat de posts sens pell ab quatre tencadors appellat Emericus super $\mathrm{III}^{\circ}$ et $\mathrm{V}^{\circ}$ decretalium.

Item altra libra scrit en paper ligat de posts ab pell verda ab quatre tencadors appellat Bartholus super tribus libris Codici.

Item altra libra scrit en paper ligat de posts sense pell ab quatre tencadors manchant ny dos appellat calim.

Item altre libra scrit en pergamí ligat de posts ab pell vermella $a b$ quatre tencadors manchant ny tres appellat Consuetuts de Catalunya cum Usaticis Barchinone.

Item altra libra scrit en pergamí ligat de posts ab pell vermella $a b$ quatre tencadors appellat constitutiones Simonis legati cardinalis.

Item altra libra scrit en pergamí ligat de posts ab pell verda hi solia haver II tencadors appellat libellus rotfredi.

Item altra libra scrit en paper ligat de posts engrutades appellat tabulam curia boich.

Item altre libra scrit en pergamí ligat de posts sens pell sens tencadors appellat summa Bernardi super decretales.

Item altra libra petit scrit en paper ligat de posts ab pell vermella ab 1 tencador appellat breves decretalium.

Item altra libra scrit en pergamí ligat de posts ab pell blancha $a b$ correges per tencador appellat instituta.

Item altra libra scrit en pergamí ligat de posts ab pell verda ab quatre tencadors apellat summa azonis.

Item altra libra en paper ligat de posts sens pell ab dos tencadors apellat Bartholus super codicem.

Item altra libra scrit en paper ligat de posts ab quatre tencadors manchan y dos appelat lecturas Bartholi super VIIII ${ }^{\circ}$ Codicis.

Item altra libra scrit en pergami ligat de posts ab pell blancha ab dos tencadors appellat Summa Azonis.

Item altra libra scrit en paper ligat de posts sens pell ab quatre tencadors appellat dominicus de sancto Geminiano super I et secundo VI libri decretalium.

Item altra libra scrit en paper ligat de posts sens pell ab quatre tencadors appellat Iohanes de Platea.

Item altra libra scrit en paper ligat de posts ab pell verda $a b$ dos tencadors appellat Bartholus super XII digesti veteris.

Item altra libra scrit en paper ligat de posts ab quatre tencadors manchan ny hun appellat repertorium ludovici de cortesiis.

Item altra libra scrit en paper ligat de posts ab pell verda e negra ab dos tencadors appellat lectura domini Jacobi rebuffi super $\mathrm{X}^{\circ}, \mathrm{XI}^{\circ}$ et $\mathrm{XII}{ }^{\circ}$ codici. 
Primo 1 libra scrit en pergamí ligat de posts ab pell verda $a b$ dos tencadors appellat ars de ypochras.

Item altra libra scrit en pergamí ligat de posts ab aluda blancha ab dos tencadors appellat fisicorum et metaphisicorum.

Item altra libra scrit en pergami ligat de posts ab coberta vermella appellat secundus evicenne.

Item altra libra scrit en pergami ligat de posts la una trencada sense tencadors appellat questiones super lecturam fisicorum.

Item altra libra de paper la mitat no es scrit ligat de posts ab pell blava ab plates appellat canonicus super libris fisicorum.

Item altra libra scrit en pergamí ab les posts trencades sens tencadors appellat Aristoteles de istoriis animalium.

Item altra libra scrit en paper ligat de posts engrutades ab pell vermella ab correges per tencadors appellat guido de istoriis troianorum.

Item altra libra scrit en paper ligat de posts engrutades appellat lectura de doctrinal.

Item altra libra appellat prosodia secundum Alexandrum de Villadei.

Item altra libra scrit en paper appellat questiones phisiologie.

Item altra libra scrit en pergami appellat ars metrificature.

Item altra libra scrit en paper appellat doctrina.

Item altra libra scrit en paper appellat lectura super libris fisicorum. Item altra libra scrit en paper appellat lectura se Albert de xexonia.

Item altra libra de paper appellat questiones super libris fisicorum.

Item altra libra de pergamí appellat abrard.

Item altra libra de paper apellat logices terminorum.

Item altra libra de pergamí apellat precia manor.

Item altra libra de pergamí appellat Alexander.

Item altra libra de pergamí appellat Gramatiga den Pestrana.

Item altra libra de pergami appellat reportationes boecii.

Item altra libra de pergamí appellat sextus metaphisices.

Item altra libra de paper appelat notes.

Item altra libra de pergamí ligat sens posts appellat ysach de elementis et Johannes ebessamett, e comença ego non iurando.

Item altra libra en pergamí glosat appellat abrart.

Item altra libra en paper appellat lectura de doctrinal.

Item altra libra de paper appellat glosa super libris físicorum.

Item altra libra de pergamí appellat textus logices Aristotelis.

Item altra libra de pergamí appellat teodoricus in cirurgia.

Item altra libra de paper appellat lectura de doctrinal vella.

Item altra libra de pergamí appellat doctrinal.

Item altra libra de paper appellat questiones posteriorum.

Item altra libra de pergami de convergencia novi et veteris sacerdotii.

Item altra libra de paper de vocables. 
Item altra libra de paper appellat questiones super metafisicha.

Item altra libra de paper appellat lectura porffirii.

Item altra libra de pergamí appellat doctrinals, dolent.

Item altra libra de pergamí appellat viaticus mundi, es de medícine.

Item altra libra de paper sisternat de pergamí appellat strodos.

Item altra libra de pergami appellat abreard sotil.

Item altra libra de pergamí appellat Prascia maior.

Item altra libra de pergamí appellat ysach, es de medicina.

Item altra libra de pergamí ab cent divines.

Item altra libra de pergamí appellat dostrinal.

Item altra libra de pergamí appellat Alexandre incompletus.

Item altra libra de pergami appellat lettera accentus.

Item altra libra de paper appellat mater de virtutibus erbarum.

Item altra libra de pergamí appellat vesprals.

Item altra libra de paper appellat distinciones primi thome.

Item altra libra de pergami appellat lectura posteriorum Aristotelis.

Item altra libra de pergamí incipit secundum pricianum.

Item altra libra en paper appellat notes.

Item altra libra en paper appellat lectura fisoloffie (sic).

Item altra libra de pergamí appellat de mirabilibus terre sancte.

Item altra libra de paper appellat.

Item altra libra de paper appellat lettera doctrinalis.

Item altra libra de pergami appellat doctrinal.

Item altra libra de paper ab posts engrutades verd, comença augeo auges.

Item altra libra de paper super libro de anima.

Item altra libra de paper appellat alexander.

Item altra libra de pergami appellat notas de mestre pecathala.

Item altra libra de pergamí appellat tractats.

Item altra libra de paper appellat questions logicals de equipolleriis.

Item altra libra de paper appellat de generatione et corruptione.

Item altra libra de pergamí appellat precia menor.

Item altra libra de paper appellat supposiciones.

Item altra libra de pergamí appellat abrard, patit.

Item altra libra de pergami appellat alexander.

Item altra libra de paper appellat lectura super literam eticorum.

Item altra libra de paper appellat super tractatibus.

Item altra libra de pergamí appellat parts.

Item altra libra de pergamí appellat epistole Pauli.

Item altra libra de pergami appellat tractats.

Item altra libra de pergami appellat ores de Leyda.

Item altra libra de pergami incipit Sancta et individuae trinitatis.

Item altra libra de pergami appellat vesprals.

Item altra libra de paper sisternat de pergamí appellat boeci de consolacio. 
Item altra libra de paper appellat notes.

Item altra libra de paper incipit qui sciencia.

Item altra libra de paper appellat fformaciones partium.

Item altra libra de paper appellat paper stupor mundi.

Item altra libra de paper appellat lectura super fisicos.

Item altra libra de paper appellat tobias.

Item VIIII ${ }^{\circ}$ alios libros de paper e V altres libras de cant.

Item altra libra part de pergami de sermons qui comença abicianus opera tenebrarum.

Quiquidem libri in renditionem comptorum de dicta hereditate et manumissoria vos facta reverendo domino Episcopo seu eius vicarii penes vos restarunt prout in vicariatus dicti domini episcopi videntem et ex alia parte restituistis et realiter tradidistis mihi publica faciencia pro baiulia burgui Sancti Petri de Gallicantu Gerunde et censualium facienciam pro hereditate et manumissoria dicti defuncti. Unde renunciando exceptioni dictorum librorum et dictorum instrumentorum non habitorum et non receptorum et doli mali et in factum actioni de predictis libris et publicis instrumentis presentem vobis facio apocham et recognitionem de recepto. Actum est hoc Gerunde die XVIII mensis septembris anno a nativitate Domini Millessimo $\mathrm{CCCC}^{\circ}$ octuagesimo. Sig + num Petri Raimundi Mut prepositi predicti qui hoc laudo et firmo.

Arxiu Històric Provincial de Girona, protocol del notari N. Cotxa, notaria 7 , núm. 133; fols. 34 a 38 .

Ací apar inventari dels ben mobles de micer Berthomeu Oristà doctor en cascun dret i prevere de capítol de la Seu de Girona que son en la sua casa qui es dins la Força apres lo castell de Gironella e tense ab lo portal de sent Christofol.

La recambra que te esguard a l'ort

Primo...

Item hun banch detras lo lit e un altre petit e una taula nova ab sos capitells.

Item una cadira e una post per tenir libres penjada en la paret i dos escambells de fust.

Item tres caxes, $\mathrm{p}^{\mathrm{a}}, 2^{\mathrm{a}}$ e $3^{\mathrm{a}}$ plenes de libres de jus contenguts $\mathrm{y}$ expressats.

(en la darrera plana del document, fol. 53):

Seguexen los libres de les tres caxes ço es $\mathrm{p}^{\mathrm{a}}, 2^{\mathrm{a}}$ e $3^{\mathrm{a}}$ 
Primo Digestus vetus, Digestus novum. Consilia Lodivici et Baldus super VI Codic.

Item Bartho. super prima parte Digest. veteris et Hisofus de bello judaico ad latus librorum.

Item Apranus De gest. romanorum translatus a Petro Candido.

Item notabilia Ludivici de Roma et Tullius in tusculanis.

Item Barturus super parte Digest. veteri sisertum petatum cum pastis coopertis coloris croci. Et lectura Bart. super Digesti novi.

Item judiciali legum et inst. et Salustius Catallinariorum Iugurta.

Item liber sermonum per dominum Bartomeum Orista compositorum et recitatorum in capella pape.

Item expendium multarum rerum.

Caxa 2a.

Primo Decretum. Petrus Jacobi super iudiciarii ordine legum.

Item gensellinus et Guillermus de monte launudo super cler.

Item Arch. super VI et abbas anticus super decretales.

Item Clementine.

Item Antonius super $\mathrm{X}^{\circ}$ libro decretalium et alie clementine cum parte rubeys.

Item glo. super septem capitulis. Et compendium tanlegre. Et postille clementinarum per dominum Nicolaum.

Item Polibius de gestis romanorum et recollecte super $\mathrm{p}^{\circ}, 2^{\circ}$ et $3^{\circ}$.

Item VI Codic. cum copertis.

Item alie recollecte iuris civilis super digesti le. $\mathrm{p}^{\circ}$ et digestum de verborum obligationibus et ff. de adquirendam her.

Item recolecte de rebus eclesie et super cert. rubric. libri VI.

Item Constituciones Tarracone et decretales et repertorium D.B. Orista.

Item Bagarotus de iudiciali ordine.

\section{Caxa 3a.}

Item hun libre de forma de full menor recollecte super libro $2^{\circ}$.

Item altre semblant que son recollectes sobre lo primer libre.

Item un altre semblant que son recollectes super VI.

Item un libre ab posts de maior full es poeta e comença es hodie.

Item un Tulli de papaer ab cuberta de pergamí. E recollectes sobre part de la Instituta.

Item recollectes sobre part del Decret ab cuvertes de pergami.

Item altre semblant ço es recollectes sobre lo $20 \mathrm{n} \mathrm{li}^{\circ}$.

Item recollectes dades a Roma e altre Tulli de officiis de papar ab cubertes de pergamí. 
Item un altre semblant que son recollectes sobre $\mathrm{V} \mathrm{li}^{\circ}$.

Item tres libres no ligats ab cuberta de pergamí e forma de ful menor no cosits.

Item un libre vermell ab platons de lautó que comença de filio prodigo.

Item les ordinacions dels censals pel S. rey fetes.

Item un breviari.

(Només he transcrit la part del document que fa referència a llibres).

Notari Antoni Arnau, notaria 3, vol. 144, fol. 51 i seg. 29 d'abril de 1488. 